\title{
Learning User Activities from Energy Demand Profiles
}

\author{
M. $\operatorname{Ros}^{1}$ M. Molina-Solana ${ }^{1}$ M.J. Martin-Bautista ${ }^{1}$ M. Delgado ${ }^{1}$ A. Vila ${ }^{1}$ \\ ${ }^{1}$ Department of Computer Science and AI. Universidad de Granada
}

\begin{abstract}
In this paper, we propose the use of energy load profiles to learn human activities. An energy load profile determines the energy consumption of an appliance during a specific interval of time. We propose the use of clustering techniques to group the different profiles according to their temporal consumption. Both Hard and Soft clustering techniques are evaluated. We have tested the method with data from REMODECE (Residential Monitoring to Decrease Energy Use and Carbon Emissions in Europe $)^{1}$ database.
\end{abstract}

Keywords: Ambient Intelligence, Modelling human activities, Energy profiles, Clustering

\section{Introduction}

Ambient Assisted Living (AAL) is a new research area that appears by applying Ambient Intelligence to the field of elderly people assistance at home. AAL has emerge as one of the most supportive to monitor human activities to support different field areas, such as, e-health, assisted-living, entertainment, and most recently energy efficiency. Its aims are to increase elders' autonomy and their home staying. Among its issues, the modelling of the human activities is becoming more prominent.

In that regard, learning human activities requires the design of dynamic systems capable of learning different types of predefined activities in real time. Mainly, those systems are based on two main concepts: actions and behaviours (or activities). We define an action as an event that is done by users at a given place and time; whereas, a behaviour is a sequence of actions that is executed in a specific interval of time. A formal definition of those concepts can be found in [1].

In general, systems able to model activities are capable of learning the relevant "actions" or "elements" for a specific behaviour $[1,2,3$, 4], determining which elements should (or not) belong to the studied activities. To do that, they need information about the user activities in the environment. That process can be seen from different points of view, depending on the sensors' localizations and usages, named wearable sensors o environmental sensors. The former ones are more focused on users' atomic activities, attached to the

\footnotetext{
${ }^{1}$ http://remodece.isr.uc.pt
}

users and able to gather any movement that they may perform. Traditionally, those methods are focused on learning and recognizing user's movements (e.g. walking, running, standing up, etc.) [5]. On the other hand, environmental sensors are capable of providing a vision of the whole scenario. These sensors are located in specific and prefixed points in the ambient, where users can interact with them without noticing. Those interactions are used by the algorithms to infer the user activities and support them. The most typical example of this kind of scenario is the Smart Environments, and more concretely, Smart Homes[6, 7].

Moreover, most of the previous approaches rely on expert information to understand the activities, either experts specify which actions should be studied or they specify the temporal period of time when the behaviour should be performed. Nevertheless, not always can we have this kind of information or, even if we have it, not always represents the users activities. General Activities are usually provided without users' own characteristics. On the other hand, in most cases, the installation of $\mathrm{Hu}$ man Activities Systems in a real scenario implies that the user have to invest some money in order to adapt the environment to the systems' requirements, for instance, installing pressure sensors in the chair to detect whether or not the user is sitting on it. Not all users are willing to incorporate to their homes devices (sensors, actuators) that can control each of their movements. Related to this drawback is the fact that the user environment cannot be changed. Systems modelling human activities are based on the Ambient Intelligence features: transparent (work unobtrusively, without users noticing the existence of devices in the environment) and ubiquitous (potentially, they can provide support any where at anytime).

New advances in the Energy field could help to overcome those drawbacks. Since the start of the Energy Efficiency Awareness, researchers have tried to accurately introduce the human factor within the models of energy loads. Some of them have put its emphasis precisely on modelling human activities since it could help in the prediction of energy requests services in housing, in order to avoid some problems like peak consumption. Several studies [8] confirm that users (and their associated behaviours) are a crucial factor in modelling energy demand (even more than the equipment itself, in some cases). 
Energy load is quite influenced by different factors, such as, weather, building infrastructure, internal working equipment, etc. Nevertheless, none of those parameters influences the building energy demand as much as the user activities inside them [9]. For instance, the expected energy demand for a user when she is cooking cannot be the same that the needed when working out at home. The energy demand required in each case is completely different, not only because the devices they are using but also to maintain the user comfort. In the energy domain, it has been proved that it is not difficult to infer the behavioural patterns of the inhabitants of a house from their energy consumption data [10].

Currently, electric companies have mechanism to monitor the electricity consumption by users at home, and even further, some of them know which devices are the one consuming the electricity. New thermostats that learn when to switch on and which temperature to set are the most prominent example $\left(\right.$ Nest thermostat ${ }^{2}$ ). Energy consumption and energy demand can be represented as time series or a graphic curve representation. As expected, the consumption included in the profile will be higher when the appliances are used, what, in most cases, means that the user was interacting with them. Therefore, studying those profiles we can learn when the user is performing particular actions.

Based on this fact, we propose a method to model user activities at residence according to their energy profiles. The method assumes that, generally, a type of behaviour is composed by a sequence of actions that are daily executed at the same interval of time[1]. For example, if someone has to start working around 9:00 everyday, we could assume that she has breakfast around 8:00, using specific devices to prepare it, such as, toaster, coffee machine or kettle. The use of those appliances can be seen as actions (e.g. she turns on the toaster), events over the devices. Thus, we propose a method capable of grouping the Energy profiles regarding temporal coincidence. Our proposal uses the Clustering technique, to be precise, $k$-means and Fuzzy $k$-means, to groups the temporal similar energy load profiles, generalizing them as part of the same behaviour.

The remainder of this paper is organized as follows. In Section 2, a background of the relevant aspects of this paper is presented, in concrete, the main relevant aspects of Modelling human activities, User activities impact on energy demand and Temporal Clustering are explained. Section 3 shows the method to learn the user activities based on Energy load profiles in depth. The experimental results are shown in Section 4, and finally, Section 5 offers the conclusion.

\footnotetext{
${ }^{2}$ https://nest.com/
}

\section{Background}

In this section, we present a brief state of the art of the most relevant areas that we combine in this approach: modelling human activities, the impact of user activities on the energy demand and time series clustering.

\subsection{Modelling human activities}

Most projects in this area focus in attendance of elders or people with special needs at smart homes, and more specifically in the modelling, recognition, and prediction of human activities, as for instance the projects TigerPlace [6], Cogknow [7], and CASAS [11].

Previous works offer studies of a wide variety of aspects regarding human activities, ranging from the underlying sensor network for user data acquisition [12], to representation [3], behaviour modelling [4], temporal aspects [13] and applications [6]. Probabilistic and uncertainty models are the most common choice in the literature, such as probabilistic sequences of objects touched by the user [12], Learning Automata[1], Hidden Markov Models (HMMs) [3], Markov Decision Processes [14] or image feature extraction techniques combined with a Naive Bayes classifier to identify the human activities [15]. A complete taxonomy of recognizing human activities is proposed in [16].

However, most of these projects depends on the reliability of the sensors and actuators installed in the environment, and on the user acceptance of those devices. In this paper, we propose the use of an alternative source of information, energy user profiles, since those profiles can determine the user actions by detecting whether or not the user is using a specific appliance.

\subsection{User activities impact on energy demand}

Modelling user activities is an emerging research area, and even more, applied to the Energy field. In the last few years, both researchers and enterprises have agreed that understating user's energy consumption is key to control the energy consumption, above all, in those buildings where users could access easily to appliances [9]. In that way, a first approach for modelling user behaviour in the context of real energy is proposed in [17], focused on learning the window opening behaviour in residential buildings. The methodology is based on a medium/long-term monitoring, developing a probabilistic approach for modelling the human behaviour related to the control of indoor environment.

The user impact on energy consumption may be measured in different levels of granularity, always depending on the type of building and its use. Rollins et al. [18] made a first approach studying the influence of users in energy requirements at 
individual appliances level. Authors aimed at understanding how users consume energy by means of specific appliances, finding its schedules of usage. Similar studies have tried to understand the effect of domestic occupancy profiles on the energy performance of a house or industry[19].

In this paper, we will try to step forward studying the influence of user at appliances levels, in order to modelling the human behaviour and supporting the daily living routines.

\subsection{Clustering of time data series}

Clustering is the unsupervised classification of patterns (observations, data items, or feature vectors) into groups (clusters)[20]. Traditionally, clustering algorithms have been divided into partitioning and hierarchical algorithms. The former one divides the data in $k$ clusters, with $k$ as an input parameter of the algorithms, whereas the later divides the scope into regions, and each cluster is contained in one of that regions. Among its advantages, clustering techniques are considered as an unsupervised learning since do no require domain knowledge and labelled data. For this reason, clustering is quite useful for pattern-analysis, grouping, decision-making, and machine-learning situations, including data mining, document retrieval, image segmentation, and pattern classification [20].

Focusing on Time series, the clustering techniques aim to partition time series data into groups based on a similarity measure or distance, obtaining a cluster in which all the time series included in it are similar, finding the most homogeneous clusters that are as distinct as possible from other clusters. Various algorithms have been developed to cluster different types of time series data [21]. Some approaches work directly with raw time series data, called raw-data-based approach, while others converts a raw time series data either into a feature vector of lower dimension or a number of model parameters called feature-based and model-based approach.

\section{Clustering energy demand profiles to identify User activities}

In this section, we propose a clustering method to find common patterns among the user energy demand profiles. The basic idea is to partition the set of time series into a specific number of clusters according to the similarity measured. Each cluster will represent a type of behaviour: a set of actions that, performed during the same period of time, have a common objective. On the other hand, each time series in the cluster represents the action that the user is performing and when she should do it.

In previous approaches, we have followed the idea that we have a monitored environment, from which we can monitor the user during any of their movements [22]. In addition, we used expert knowledge

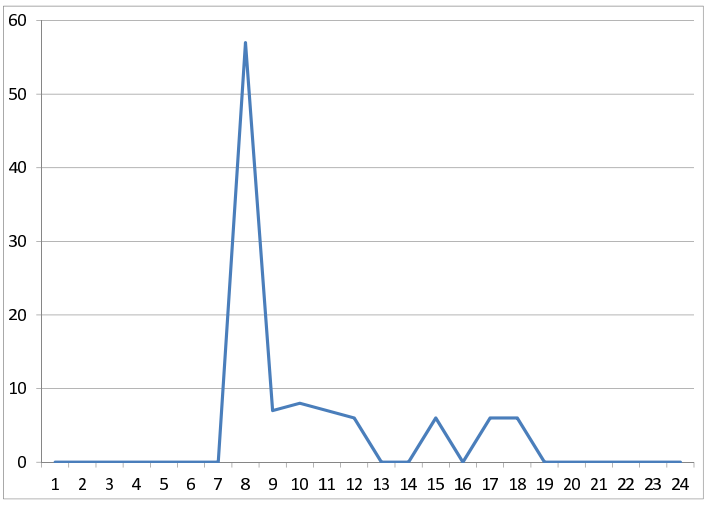

Figure 1: Example of a Daily load profile for a Kettle

to identify activity periods at home in order to study a specific piece of the Stream Data generated from the monitoring process $[1,13]$. Even though these kind of assumptions are the most common in the AAL research field, we cannot always rely on having the expert knowledge or having a monitored house. In order to overcome these possible limitations, we propose a method that uses alternative information to determine whether or not an user is doing something at their home, concretely, energy load profiles of each appliance at home. Generally speaking, Energy Load Profile represents the electricity usage for appliances related to different building uses and typologies. They provide the information about when a specific device is used, for how long and the intensity of its use. An example of energy load profiles is shown in Figure 1, which represents the daily load profile for a Kettle. As can be seen, although the Kettle may be used during the whole day, it is used with more intensity and frequency from 07:00 to 09:00, what could be generalized as it being part of the 'having breakfast' routine.

In general, profiles represent realistic detailed information, since they mean the end-users interaction with the energy equipment and consuming devices. Although the generation of the energy demand profiles is not always direct (as proven by the numerous studies about the profile generation process [19]), in this paper we assume that we have those profiles ready to be used in our system.

As a Energy Load Profile represents a temporal evolution of the electricity demand, we can represent them as time series. A time series is a sequence of real values collected at predefined intervals of time or prefixed time. We can represent a time series as an ordered set of $n$ real values $\left(Y=y_{1}, y_{2}, \ldots ., y_{n}\right)$, where $n$ is the length of $Y$, and $y_{i}$ is the real valued number of $Y$ at timestamp $i$.

We should remember that when two time series are compared they should be normally sampled at the same interval, and their length (or number of time points) be the same, if possible.

Once we have the Energy Load Profiles as time series, we are prepared to apply Time-Series clus- 
tering to them. We propose to use the well-known clustering method of $k$-means [20], although we plan to apply other clustering techniques in the near future.

$k$-means is based on the concept of the minimization of a fitness function, traditionally, defined as the distance between two elements, or in our case, two time series:

Definition 1 ( $K$-means algorithm [21]). Given $n$ patterns $x_{k} \mid k=1, \ldots, n, k$-means determine $k$ clusters centers $v_{i} \mid i=1, \ldots, k$, by minimizing the objective function given as

$$
\min J_{i}(U, V)=\sum_{i=1}^{k} \sum_{c=1}^{n} u_{i c} d\left(x_{k}, v_{i}\right)
$$

where $u_{i k} \in 0,1 \forall i, k, \sum_{i=1, k} u_{i k}=1 \forall k$, and $d(x, y)$ is the distance measure.

$K$-means assigns to every cluster a centroid. Each element will be included in the cluster whose centroid is more similar (computed by a similarity measure defined according to the problem's domain). In every iteration, the centroid is updated by taking into account the new elements included in the cluster.

The $k$-means algorithm is classified as Hard clustering: any profile $X$ either is or is not part of a particular cluster. However, in our domain, some actions (profiles) may be part of different activities. For instance, turning television on and sitting on a chair could be part of both having breakfast and watching television. Therefore, we need a method capable of performing Soft clustering over the different profiles: a profile $X$ might belong to more than one cluster, with associated membership values. The membership value will help to determine the strength of the association between the profile and the cluster.

To be precise, we propose the use of the fuzzy version of the k-means algorithm: Fuzzy $k$-means $(\mathrm{FCM})[23]$.

Definition 2 (Fuzzy k-means algorithm [21]). Given $n$ patterns $x_{k} \mid k=1, \ldots, n, k$-means determine $k$ clusters centers $v_{i} \mid i=1, \ldots, k$, by minimizing the objective function given as

$$
\min J_{i}(U, V)=\sum_{i=1}^{k} \sum_{c=1}^{n}\left(\mu_{i k}\right)^{2} d\left(x_{k}, v_{i}\right)
$$

where $u_{i k} \in 0,1 \forall i, k, \sum_{i=1, k} u_{i k}=1 \forall k$, and $d(x, y)$ $i s$ the distance measure.

Since similarity is fundamental to the definition of a cluster, a measure of the similarity between two time series has to be defined. Several methods, such as Dynamic time warping (DTW) or others, have been tested to select the most adequate similarity measure in our context. Finally, although the use of Euclidean Distance in the context of time series is controversial, we have selected to use it since it facilitates to compare two (time-dependent) sequences where the sequences has a linear (elastic) alignment. Using Euclidean distance, we can calculate which elements are used during the same interval of time through matching similar shapes of phase in the time axis. The Euclidean distance is computed as follows:

Definition 3 (Euclidean distance). Let $x_{i}$ and $y_{j}$ be a P-dimensional vector of values. We compute the Euclidean distance as:

$$
d\left(x_{i}, y_{j}\right)=\sqrt{\sum_{K=1}^{P}\left(x_{i k}-y_{j k}\right)^{2}}
$$

\section{Experiment and Discussion}

\subsection{Experimental settings}

To run our experiments, we use the REMODECE (Residential Monitoring to Decrease Energy Use and Carbon Emissions in Europe $)^{3}$ database. This database stores characterizations of residential electricity consumption by end-user and by country, including Central and Eastern European Countries.

Among other type of information, we can retrieve Daily average load curves for specific appliances and projects, collected in the REMODECE project. Those curves are divided into 24 hours, with one hour intervals. Every point in the profile represents the Power consumed by the appliances during that interval.

As example of the data used in the experiment, Figure 2 shows some Daily load profiles for a specific project, included in the REMODECE database, Remodece $\mathrm{Fr}$ in France (2006-2008) for some appliances. Notice that we have normalized the values of Power (Watts) in order to compare all the appliances in the same conditions.

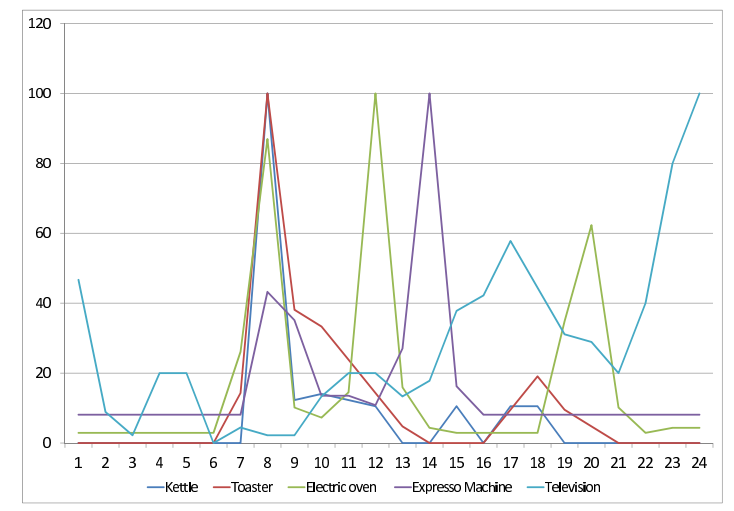

Figure 2: Daily average load curve for Remodece Fr in France (2006-2008) Project, for Having Breakfast

Although REMODECE database contains data from different projects and appliances, they are not

\footnotetext{
${ }^{3}$ http://remodece.isr.uc.pt
} 


\begin{tabular}{|c|c|}
\hline Project & Appliances \\
\hline Remodece Fr & $\begin{array}{c}\text { DVD Player, Television, } \\
\text { Home Cinema, Laptop, } \\
\text { Bread Maker,Kettle, } \\
\text { Espresso Machine, Toaster, } \\
\end{array}$ \\
\hline Remodece Cz & Television, Laptop, \\
& Kettle, Espresso Machine, \\
& Toaster, Dish Washer, \\
& Microwave \\
\hline Remodece It & DVD Player, Television, \\
& Home Cinema, Laptop, \\
& Bread Maker,Kettle, \\
& Espresso Machine, Toaster, \\
& Dish Washer, Microwave \\
\hline
\end{tabular}

Table 1: Appliances studied for each project during (2006-2008)

complete, since we can find combinations of those two elements that do not contain stored data. In order to compare correctly the results obtained, we have selected only those examples that we can consider complete: they have data for each appliance that we studied. The different combinations used in this test are collected in Table 1.

According to Remodece profiles and expert knowledge, three types of behaviour could be identified from data: To have breakfast $\left(B_{1}\right)$, To watch television $\left(B_{2}\right)$ and To wash the dishes $\left(B_{3}\right)$.

In order to execute our experiments, we have used the matlab functions ${ }^{4} \mathrm{kmeans}$ and $\mathrm{fcm}$ used for $k$ means clustering and FCM clustering, respectively, to partition the profiles data into $k$ clusters.

\subsection{Results and discussion}

Over this dataset, we have tested the performance of both $k$-means and fuzzy k-means algorithms. We have run the experiments for $k=3$ (the number of behaviours that we expected to learn: To have breakfast $\left(B_{1}\right)$, To watch television $\left(B_{2}\right)$ and To wash the dishes $\left(B_{3}\right)$. Table 2 summarizes the results for k-means and Fuzzy k-means for each used dataset. We organize the results for each behaviour, applied algorithm and number of clusters as percentages in four categories: True positives (TP) are positive profiles correctly labelled in the type of behaviour. False positives (FP) refer to negative profiles incorrectly labelled as part of the behaviour. True negatives (TN) correspond to negative profiles correctly labeled as non part of the behaviour. Finally, false negatives (FN) refer to positive profiles incorrectly labelled as non part of the behaviour.

As seen from Table 2, a $100 \%$ score in most of the positive and negative patterns was obtained, meaning that the profile where correctly classified by the system. In addition, we obtained a $0 \%$ of most of false positive and false negative patterns,

\footnotetext{
${ }^{4}$ Mathworks.http://www.mathworks.com
}

what means that those profiles that should not belong to the behaviour are correctly categorized as non-part. Also, notice that, although we could assume that having less profiles could make easier the clustering process, we obtained good results with the Remodece It dataset composed by 10 different profiles. On the other hand, we would like to remark that the best results are obtained for the To have breakfast $\left(B_{1}\right)$ behaviour. The reason of that fact is that its temporal execution is quite different from the rest, and it cannot happen in any moment of the day. This is a problem that may be solved combining the similarity measures.

Focusing on flexibility instead of accuracy, we can affirm that $F C M$ provides better results than $k$-means, since FCM profiles can be included in different clusters. The coffee machine is an example of this situation. In the Remodece It dataset, using the $k$-means algorithm the coffee machine is excluded from the To have breakfast $\left(B_{1}\right)$ (see score $75 \%$ from 2). However, using $F C M$ that profile is included, although not with a $100 \%$ membership degree.

\section{Conclusions}

In this paper, we have proposed a method to group Energy load profiles in order to obtain the relevant actions of a specific type of behaviour. Energy load profiles provide information about the energy consumption of appliances during a period of time. This fact allows us to hypothesize that higher consumption means the user is using that appliance. We analysed that information, found relationships between different profiles (appliances, actions) and generalized them as particular behaviours. To achieve these objectives, we have proposed the use of time series clustering techniques, $k$-means and Fuzzy $k$-means with the Euclidean distance, and tested them.

In this domain, we have proved that Fuzzy $k$ means produces more flexible results when some actions belonging to different activities are expected. However, as anticipated, the $k$-means algorithm is more accurate because it is capable to distinguish between the true relevant actions and those that might be done during the performance of a behaviour, but are not main actions.

As future work, we aim to provide a complete semantic description to the Energy Load Profiles and extracted clusters (behaviours), together with a recognition system capable of identifying user actions in real time and provide support to perform them. As well, we would like to make users understand their own habits, routines, from the energy point of view, in order to raise user consciousness of the energy squander and suggest some changes in their behaviour that could help to manage and reduce the energy at home. 


\begin{tabular}{|c||c||c||c||c||c||c|}
\hline Dataset & Behaviour & Algorithm & \% TP & \% TN & \% FP & \% FN \\
\hline Remode FR & To have & $k$-means & 100,00 & 100,00 & 0,00 & 0,00 \\
& breakfast $\left(B_{1}\right)$ & $F C M$ & 100,00 & 66,67 & 33,33 & 0,00 \\
\hline Remode FR & To watch & $k$-means & 75,00 & 100,00 & 0,00 & 25,00 \\
& television $\left(B_{2}\right)$ & $F C M$ & 100,00 & 100,00 & 0,00 & 0,00 \\
\hline Remode FR & To wash the & $k$-means & 100,00 & 50,00 & 50,00 & 0,00 \\
& dishes $\left(B_{3}\right)$ & FCM & 100,00 & 66,70 & 33,30 & 0,00 \\
\hline \hline Remodece Cz & To have & $k$-means & 75,00 & 100,00 & 0,00 & 25,00 \\
& breakfast $\left(B_{1}\right)$ & FCM & 100,00 & 66,67 & 33,33 & 0,00 \\
\hline Remodece Cz & To watch & $k$-means & 100,00 & 100,00 & 0,00 & 0,00 \\
& television $\left(B_{2}\right)$ & FCM & 100,00 & 100,00 & 0,00 & 0,00 \\
\hline Remodece Cz & To wash the & $k$-means & 100,00 & 0,00 & 100,00 & 0,00 \\
& dishes $\left(B_{3}\right)$ & FCM & 100,00 & 100,00 & 0,00 & 0,00 \\
\hline \hline Remodece It & To have & $k$-means & 75,00 & 50,00 & 50,00 & 25,00 \\
& breakfast $\left(B_{1}\right)$ & FCM & 100,00 & 70,00 & 30,00 & 0,00 \\
\hline Remodece It & To watch & $k$-means & 75,00 & 100,00 & 0,00 & 25,00 \\
& television $\left(B_{2}\right)$ & FCM & 100,00 & 90,00 & 10,00 & 0,00 \\
\hline Remodece It & To wash the & $k$-means & 100,00 & 50,00 & 50,00 & 0,00 \\
& dishes $\left(B_{3}\right)$ & FCM & 100,00 & 70,00 & 30,00 & 0,00 \\
\hline
\end{tabular}

Table 2: Accuracy of $k$-means and FCM for the studied behaviour

\section{Acknowledgments}

This work was partially funded by the Spanish Ministry of Economy (project TIN2012-30939) and the European Union (Energy IN TIME project, grant agreement no. 608981). The authors also thanks IFSA's reviewers for their valuable comments on earlier versions of this work.

\section{References}

[1] María Ros, Manuel P. Cuéllar, Miguel Delgado, and Amparo Vila. Online recognition of human activities and adaptation to habit changes by means of learning automata and fuzzy temporal windows. Inf. Sci., 220:86-101, 2013.

[2] Hani Hagras, Victor Callaghan, Martin Colley, Graham Clarke, Anthony PoundsCornish, and Hakan Duman. Creating an ambient-intelligence environment using embedded agents. IEEE Intelligent Systems, 19(6):12-20, 2004.

[3] D.H. Wilson, D. Wyaat, and M. Philipose. Using context history for data collection in the home. In Pervasive, volume 3468, 2005.

[4] F. Doctor, H. Hagras, and V. Callaghan. A fuzzy embedded agent-based approach for realizing ambient intelligence in intelligent inhabited environments. IEEE Transactions on Systems, Man, and Cybernetics, Part A, 35(1):55$65,2005$.
[5] J. Pärkkä, M. Ermes, P. Korpipää, J. Mäntyjärvi, J. Peltola, and I. Korhonen. Activity classification using realistic data from wearable sensors. IEEE Transactions on Information Technology in Biomedicine, 10(1):119128, 2006. cited By 216.

[6] M.J. Rantz, M. Aud, G. Alexander, D. Oliver, D. Minner, M. Skubic, J. Keller, Z. He, M. Popescu, G. Demiris, , and S. Miller. Tiger place: An innovative educational and research environment. In $A A A I$ in Eldercare: New Solutions to Old Problems, 2008.

[7] C.D. Nugent, M. D. Mulvenna, F. Moelaert, B. Bergvall-Kereborn, F. Meiland, D. Craig, R. Davies, A. Reinersmann, M. Hettinga, AL. Andersson, R-M. Dries, and J. Bengtsson. Home-based assistive technologies for people with mild dementia. In Pervasive Computing for Quality of Life Enhancement, volume 4541, pages 63-69, 2007.

[8] K. Jessoe and D. Rapson. Knowledge is (less) power: Experimental evidence from residential energy use. Working Paper 18344, National Bureau of Economic Research, August 2012.

[9] Lee Schipper and Stephen Meyers. Energy Efficiency and Human Activity: Past Trends, Future Prospects. Cambridge University Press, 2005.

[10] Patrick McDaniel and Stephen McLaughlin. Security and privacy challenges in the smart grid. IEEE Security and Privacy, 7(3):75-77, 
2009.

[11] Parisa Rashidi, Student Member, Diane J. Cook, Lawrence B. Holder, and Maureen Schmitter-edgecombe. Discovering activities to recognize and track in a smart environment. IEEE Trans. on Knowledge and Data Engineering, to appear:14, 2010.

[12] M. Philipose, K. P. Fishkin, M. Perkowitz, D. J. Patterson, D. Fox, H. Kautz, and D. Hahnel. Inferring activities from interactions with objects. IEEE Pervasive Computing, 3(4):5057, 2004.

[13] María Ros, Miguel Delgado, and Amparo Vila. Fuzzy method to disclose behaviour patterns in a tagged world. Expert Systems with Applications, In Press, Corrected Proof, 2010.

[14] J. Boger, J. Hoey, P. Poupart, C. Boutilier, G. Fernie, and A. Mihailidis. A planning system based on markov decision processes to guide people with dementia through activities of daily living. IEEE Trans Inf Technol Biomed, 10(2):323-333, 2006.

[15] Ross Messing, Chris Pal, and Henry Kautz. Activity recognition using the velocity histories of tracked keypoints. In Computer Vision, 2009 IEEE 12th International Conference on, pages $104-111$, sep. 2009.

[16] Natalia Díaz Rodríguez, Manuel P. Cuéllar, Johan Lilius, and Miguel Delgado Calvo-Flores. A fuzzy ontology for semantic modelling and recognition of human behaviour. Knowl.-Based Syst., 66:46-60, 2014.

[17] Valentina Fabi, RuneVinther Andersen, StefanoP. Corgnati, and BjarneW. Olesen. A methodology for modelling energy-related human behaviour: Application to window opening behaviour in residential buildings. Building Simulation, 6(4):415-427, 2013.

[18] S. Rollins and N. Banerjee. Using rule mining to understand appliance energy consumption patterns. In Pervasive Computing and Communications (PerCom), 2014 IEEE International Conference on, pages 29-37, March 2014.

[19] Violeta Motuziene and Tatjana Vilutiene. Modelling the effect of the domestic occupancy profiles on predicted energy demand of the energy efficient house. Procedia Engineering, 57(0):798 - 807, 2013. Modern Building Materials, Structures and Techniques.

[20] Anil K. Jain and Richard C. Dubes. Algorithms for Clustering Data. Prentice-Hall, Inc., Upper Saddle River, NJ, USA, 1988.

[21] T. Warren Liao. Clustering of time series dataa survey. Pattern Recognition, 38(11):1857 1874, 2005.

[22] Miguel Delgado, María Ros, and M. Amparo Vila. Correct behavior identification system in a tagged world. Expert Syst. Appl., 36(6):9899-9906, 2009.
[23] James C. Bezdek. Pattern Recognition with Fuzzy Objective Function Algorithms. Kluwer Academic Publishers, Norwell, MA, USA, 1981. 